\title{
Microscopy
}

\section{Approaches to 3D Printing Teeth from X-Ray Microtomography}

\begin{tabular}{|r|l|}
\hline Journal: & Journal of Microscopy \\
\hline Manuscript ID & Draft \\
\hline Wiley - Manuscript type: & Themed Issue Paper \\
\hline Date Submitted by the Author: & n/a \\
\hline Complete List of Authors: & $\begin{array}{l}\text { Cresswell-Boyes, Alexander; Queen Mary, University of London, Barts and } \\
\text { The London, School of Medicine and Dentistry } \\
\text { Barber, Asa; London South Bank University, School of Engineering } \\
\text { Tatla, Amo; GlaxoSmithKline, Oral Health, Research \& Development } \\
\text { Mills, David; Queen Mary, University of London, Barts and The London, } \\
\text { School of Medicine and Dentistry } \\
\text { Davis , Graham; Queen Mary, University of London, Barts and The London, } \\
\text { School of Medicine and Dentistry }\end{array}$ \\
\hline Keywords: & $\begin{array}{l}\text { X-Ray Microtomography, Additive Manufacturing, Dental Materials, Pre- } \\
\text { Clinical Teaching }\end{array}$ \\
\hline
\end{tabular}




\title{
Approaches to 3D Printing Teeth from X-Ray Microtomography
}

\author{
A.J. Cresswell-Boyes ${ }^{*}$, A.H. Barber ${ }^{\dagger}$, D. Mills ${ }^{*}$, A. Tatla ${ }^{\ddagger} \&$ G.R. Davis ${ }^{*}$ \\ *Dental Physical Sciences, Institute of Dentistry, Francis Bancroft Building, Queen Mary \\ University of London, Mile End Road, London, E1 4NS, UK. \\ ${ }^{\dagger}$ School of Engineering, London South Bank University, Borough Road, London, SE1 0AA. \\ "GlaxoSmithKline, St George’s Avenue, Weybridge, KT13 0DE, UK.
}

Key words. X-Ray Microtomography, Additive Manufacturing, Dental Materials, PreClinical Teaching.

\begin{abstract}
Artificial teeth have several advantages in pre-clinical training. The aim of this study is to 3D print accurate artificial teeth using scans from x-ray microtomography (XMT). Extracted and artificial teeth were imaged by the in-house XMT MuCAT-2 system at Queen Mary at $90 \mathrm{kV}$ and $40 \mathrm{kV}$ respectively, to create detailed high contrast scans. The dataset is then viewed in Drishti, where both internal and external meshes can be exported to 3D modelling software (Meshlab), for modification before finally exporting to a slicing program (Cura) for printing. After appropriate parameter setting, the printer deposits material in specific locations layer by layer, to create a 3D model. Scans are trimmed to ensure a clean model which is then imported into the slicing software, where layer height could be determined to replicate the high resolution that was seen in the XMT scans. The model was then printed in two different materials (polylactic acid and thermoplastic elastomer), changing settings dependent on the material. A multi-material print was created to show the different physical characteristics between enamel and dentine.
\end{abstract}

\section{Introduction}

Within dental education, there is a distinct need for hands-on pre-clinical training before treatment of a patient (Qualtrough et al., 1999). Throughout the years, numerous techniques have been created to teach students to perform different dental treatments. Artificial teeth are widely used and replicated with transparent resins in which the pulp chamber and root canals are distinguishable with different colours (Nassri et al., 2008). Despite this, extracted human teeth remain one of the most popular techniques used during pre-clinical endodontic training. Both techniques have advantages and disadvantages, with the common practice being a combination of artificial root canal models and extracted human teeth during pre-clinical training (Dummer, 1991).

The disadvantages of extracted teeth include; the possibility of cross-infection, being potentially infectious to students; the unavailability of these teeth and anatomical variability of extracted teeth, meaning valid assessment for students is not uniform (Tchorz et al., 2015).

The benefits of artificial teeth is that they do not have a risk of infection, are available in large quantities, allow for validated assessment through their uniformity (Bitter et al., 2016), can be tailored to offer anatomical challenges, and can be three-dimensionally (3D) printed into training models for simulation-based medical education (SBME) (San Diego et al., 
2013). However, based on previous studies, students reported artificial teeth as unsatisfactory because of a perceived lack of realism of these teeth, offering them a non-realistic simulation to practice on, compared to natural teeth. Despite the reported difficulties, students recognised the advantages and suggested improvements, such as varying geometry, to include real-life imperfections of teeth such as caries (Al-Sudani \& Basudan, 2016).

Previous literature studies have shown the ability to convert tomography files into viable 3D printed training models (O'Brien et al., 2016), in Longfield, et. al. (2015) CT scans were taken of 6-month-old patient's temporal bone for a low-cost training method in paediatric surgery (Longfield et al., 2015). In this study, we present a method of collecting real-world biological data and converting into a 3D model, using open source software. The emphasis at this stage of the study is to focus on the geometry of the tooth, with future work looking into mimicking the mechanical properties of the tooth.

\section{Materials and Methods}

\section{X-Ray Microtomography}

A natural mandibular first molar and a plastic replica (Fábrica de Sorrisos, Brazil), were selected as specimens to demonstrate the transition from XMT to 3D print. The specimens were scanned using the 'in-house' TDI (time delay integration) X-ray microtomography scanner at Queen Mary University of London, developed by Davis and Elliott (2003). The system called MuCAT-2 uses charge-coupled detector (CCD) camera (Spectral Instruments, USA) with a $60 \mu \mathrm{m}$ thick columnar caesium iodide scintillator (Applied Scintillation Technologies, Ltd., UK) (Davis \& Elliott, 2003; Davis et al., 2013). The natural tooth was scanned at $15 \mu \mathrm{m}$ at $90 \mathrm{kV} 180 \mu \mathrm{A}, 1503$ projections were taken within 3 blocks $^{1}$, whereas the plastic tooth was scanned at $40 \mathrm{kV}, 405 \mu \mathrm{A}, 675$ projections within a single block. Time taken for the scans was $26 \mathrm{hrs} 52 \mathrm{mins}$ and $5 \mathrm{hrs} 8 \mathrm{mins}$ respectively. The projections were reconstructed using a modified Feldkamp cone-beam back-projection algorithm (Feldkamp et $a l ., 1984)$. The files produced are loaded and trimmed to produce single-byte voxel data containing only the cuboid of interest.

\section{Visualisation}

Tomview (Version 1.1; Prof. G. R. Davis, QMUL, 2003-2017) is used to view 2D slices through the trimmed volume $(*$.tom file) in any of the three orthogonal planes (XY, XZ and YZ). Tomview can also create a metadata file (*.nc file) compatible with Drishti (Version 2.6.3; ANU Vizlab, 2016), which is a multi-platform, open source volume exploration and presentation tool, written for visualising tomography datasets (*.nc). Using Drishti, a mesh is generated in the form of a polygon file format (*.ply), which is opened using Meshlab (Version 2016.12; ISTI-CNR, 2016). Using Meshlab, the 3D mesh can be modified and manipulated, removing any unwanted or excess material or imperfections.

\section{D Printing}

The model file was exported as a *.stl file format with $\mathrm{ASCII}^{2}$ coding into Cura (Version 2.3.1; Ultimaker, 2016). Cura is a $3 \mathrm{D}$ printer slicing application, in which parameters are set

\footnotetext{
${ }^{1}$ Area of view for the camera of 501 slices and 675 slices for the extracted and artificial tooth, respectively.

${ }^{2}$ American Standard Code for Information Interchange.
} 
and a 3D visualisation of each layer is provided. This information is exported into a G-code file format and fed into the 3D printer. Wanhao Duplicator i3 (Wanhao, China) an opensource fused deposition modelling (FDM) 3D printer, reads the numerical control programming language of the G-code to deposit a determined amount of material in a specific location. Modifications were made to the printer in the form of a Bowden extruder (Landry, 2016) (Flexion ${ }^{\mathrm{TM}}$, Diabase Engineering, USA), designed to increase the resolution of the Wanhao Duplicator i3 from $60 \mu \mathrm{m}$ to $50 \mu \mathrm{m}$. Filaments used for the printing include white polylactic acid (PLA) (3D Prima, Sweden), and flexible thermoplastic elastomer (TPE) (FFF World, Spain).

\section{Results}

Figs. 1-2 were chosen to illustrate the variety of images obtainable from the reconstructed volume datasets. Further details of the specimens are given in the Figure captions. Fig. 3. demonstrates the 3D surface rendering that is achievable in the open-source software.

Three models were produced to demonstrate the ability to convert tomography files to 3D prints. Two prints made from PLA, for both natural and artificial (Fig. 4(a)), the third print was an assembled model made from two different materials, PLA for the enamel and TPE for the dentine and pulp cavity (Fig. 4(b)).

\section{Discussion}

The non-destructive 3D imaging of teeth can provide a basis for SBME, using the data for the teaching of dental morphology, operative dentistry and endodontics (Dowker et al., 1997). Combining this technology with recent advances in modelling and computational power, the ability to manipulate biological datasets opens a new way of teaching. This could be further enhanced by using virtual reality/augmented reality for SBME, in which sensory feedback is received from simulated environments (Dowker et al., 1997; Wang et al., 2015). However, virtual reality is still in its infancy stage and has only recently become available in the consumer market, unlike 3D printing with desktop printers; now widely available.

XMT produces a high-resolution scan of the teeth, both extracted and artificial, which were easily converted into workable 3D models using multiple software (Drishti, Meshlab). Scans were trimmed to remove excess material, to ensure a clean model was produced. The specimens' datasets can be uploaded to a free access database for downloading to be used in $3 \mathrm{D}$ printing (websites such as Thingiverse ${ }^{3,4}$ (www.thingiverse.com)) and/or virtual reality (Sketchfab (www.sketchfab.com)). Both data collected from extracted teeth and artificial teeth showed the internal and external geometry, with the artificial tooth being scanned as a comparison to show whether the anatomy differs from natural teeth. In the example of the artificial tooth, the internal geometry showed differences in the structure between the enamel and dentine with different types of resin used to distinguish this (Fig. 2).

Drishti allows the isolation of different meshes, such as; enamel, dentine, and pulp. The isolation of different meshes allowed for the ability to print each structure separately and with different materials. With Meshlab, the meshes can be edited, changing the dimensions, and

\footnotetext{
${ }^{3}$ Extracted tooth; www.thingiverse.com/thing:2770645

${ }^{4}$ Artificial tooth; www.thingiverse.com/thing:2770647
} 
changing the number of triangles and vertices, which in turn alters the resolution of the model and the size of the model file. This combination of programs proved effective in altering the tooth datasets and could be a possible pathway to use in future conversion of XMT data to *.stl files.

Using Cura as the slicing program, allows finite control over the final product, as more parameters can be set and changed compared to other slicing programs. Cura is also compatible with most commercially available 3D printers, making it an ideal choice. Using the XMT data of the artificial tooth, replicas were produced using white PLA which contained both internal and external structures. The choice of only using one material to recreate the artificial tooth was to prove the concept, not to entirely re-create the commercial product. In reality there would be no requirement for 3D printing the artificial tooth, but for the study, to evaluate the ability to convert data into a model. The XMT data of the extracted tooth was used to create various models using white PLA. The models proved that it was possible to use a cost-effective 3D printer and produce from XMT data, physical 3D models, but at lower resolution, with the MuCAT-2 system having a resolution of around $13 \mu \mathrm{m}$, the Wanhao Duplicator 33 (with Flexion ${ }^{\mathrm{TM}}$ extruder) can only produce a resolution of around 50 $\mu \mathrm{m}$, so the high definition was not captured. The ability to create multiple meshes for the internal anatomy was taken advantage of, as a multi-material print was created, using PLA as the enamel, and the flexible TPE as the dentine and pulp, to primitively demonstrate the different mechanical properties of real enamel and dentine. The use of dual extrusion would allow for a simultaneous print using multiple materials. However, the print made of different parts allows for dismantling which could be a valuable tool in teaching students tooth anatomy especially the different structures that make up a tooth.

\section{Conclusion}

We have demonstrated that by using various open-source programs, it is possible to recreate 3D models from tomography files in particular XMT, allowing for high-resolution nondestructive scans of biological specimens. This could prove to be invaluable for SBME, as the rise in availability of desktop 3D printers for home and lab continues, along with freeaccess databases. Future work for this study will look into the mimicking the properties of natural teeth, and the possibility of recreating this with the use of 3D printing. 


\section{References}

Al-Sudani, D. I., \& Basudan, S. O. (2016). Students' Perceptions of Pre-Clinical Endodontic Training with Artificial Teeth Compared to Extracted Human Teeth. European Journal of Dental Education. doi:10.1111/eje.12223

Bitter, K., Gruner, D., Wolf, O., \& Schwendicke, F. (2016). Artificial Versus Natural Teeth for Preclinical Endodontic Training: a randomized controlled trial. Journal of Endodontics, 42(8).

Davis, G. R., \& Elliott, J. C. (2003). High Definition X-ray Microtomography using a Conventional Impact X-ray Source. Journal de Physique IV (Proceedings), 104(1), 131-134.

Davis, G. R., Evershed, A. N. Z., \& Mills, D. (2013). Quantitative High Contrast X-Ray Microtomography for Dental Research. Journal of Dentistry, 41, 475-482.

Dowker, S. E. P., Davis, G. R., Elliott, J. C., \& Wong, F. S. L. (1997). X-ray microtomography: 3-dimensional imaging of teeth for computer-assisted learning. European Journal of Dental Education, 1, 61-65.

Dummer, P. M. (1991). Comparison of Undergraduate Endodontic Teaching Programmes in the United Kingdom and in some dental schools in Europe and the United States. International Endodontic Journal, 24(1), 169-177.

Feldkamp, L. A., Davis, L. C., \& Kress, J. W. (1984). Practical Cone-Beam Algorithm. Journal of the Optical Society of America A-Optics Image Science and Vision, 1(6), 612-619.

Landry, T. (2016, 15th March 2016). Extruders 101: A crash course on an essential componenet of your 3D printer. Retrieved from www.matterhackers.com/articles/extruders-101:-a-crash-course-on-an-essentialcomponent-of-your-3d-printer

Longfield, E. A., Brickman, T. M., \& Jeyakumar, A. (2015). 3D Printed Pediatric Temporal Bone: A Novel Training Model. Otology \& Neurotology, 36(5), 793-795.

Nassri, M. R., Carlik, J., da Silva, C. R., Okagawa, R. E., \& Lin, S. (2008). Critical Analysis of Artificial Teeth for Endodontic Teaching. Journal of Applied Oral Science, 16(1), 43-49.

O'Brien, E. K., Wayne, D. B., Barsness, K. A., McGaghie, W. C., \& Barsuk, J. H. (2016). Use of 3D Printing for Medical Education Models in Transplantation Medicine: a Critical Review. Current Transplantation Reports, 3, 109-119.

Qualtrough, A. J., Whitworth, J. M., \& Dummer, P. M. (1999). Preclinical Endontology: an international comparison. International Endodontic Journal, 32(1), 406-414.

San Diego, J. P., Newton, T., Quinn, B. F. A., Cox, M. J., \& Woolford, M. J. (2013). Levels of Agreement Between Student and Staff Assessments of Clinical Skills in Performing Cavity Preparation in Artificial Teeth. European Journal of Dental Education. doi:doi: 10.1111/eje.12059

Tchorz, J. P., Brandl, M., Ganter, P. A., Karygianni, L., Polydorou, O., Vach, K., . . . Altenburger, M. J. (2015). Pre-Clinical Endodontic Training with Artificial Instead of Extracted Human Teeth?: does the type of exercise have an influence on clinical endodontric outcomes? International Endodontic Journal, 48, 888-893.

Wang, D., Li, T., Zhang, Y., \& Hou, J. (2015). Survey on Multisensory Feedback Virtual Reality Dental Training Systems. European Journal of Dental Education. doi:10.1111/eje.12173 


\section{List of Figures}

Fig. 1. Slices of natural mandibular first molar (a) mesiodistal section, exposing the buccal and lingual aspect of the pulp cavity, (b) buccolingual section, exposing the medial and distal aspect of the pulp cavity, (c) transverse section midroot and occlusal.

Fig. 2. Slices of artificial mandibular first molar (a) mesiodistal section, exposing the buccal and lingual aspect of the pulp cavity, (b) buccolingual section, exposing the medial and distal aspect of the pulp cavity, (c) transverse section trunk and occlusal.

Fig. 3. Rendered surface images of both natural and artificial mandibular first molar (a) viewed in Drishti, (b) viewed in Autodesk Meshmixer.

Fig. 4. 3D printed mandibular first molar (a) both natural and artificial molar in PLA, (b) natural molar in multiple materials, PLA for enamel, TPE for dentine and pulp cavity.

Fig. 5. Screenshots of the open-source software used to convert tomography files to 3D print (a) rendering of 3D surface in Drishti, (b) rendering of 3D surface in Meshlab, with the ability to manipulate file to desired final product, (c) slicing view in Cura, with the ability to edit layer settings. 


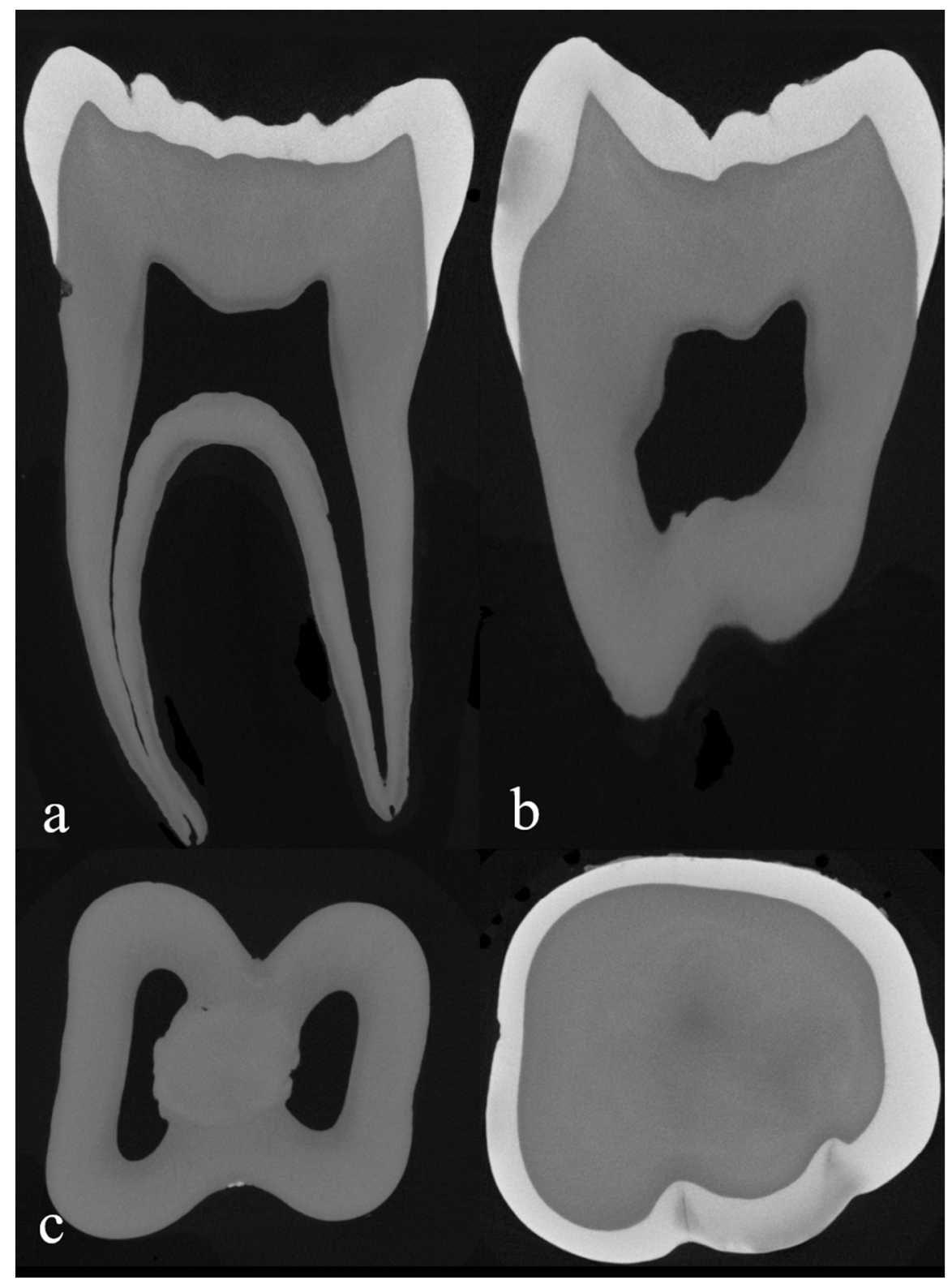

Fig. 1. Slices of natural mandibular first molar (a) mesiodistal section, exposing the buccal and lingual aspect of the pulp cavity, (b) buccolingual section, exposing the medial and distal aspect of the pulp cavity, (c) transverse section midroot and occlusal. 


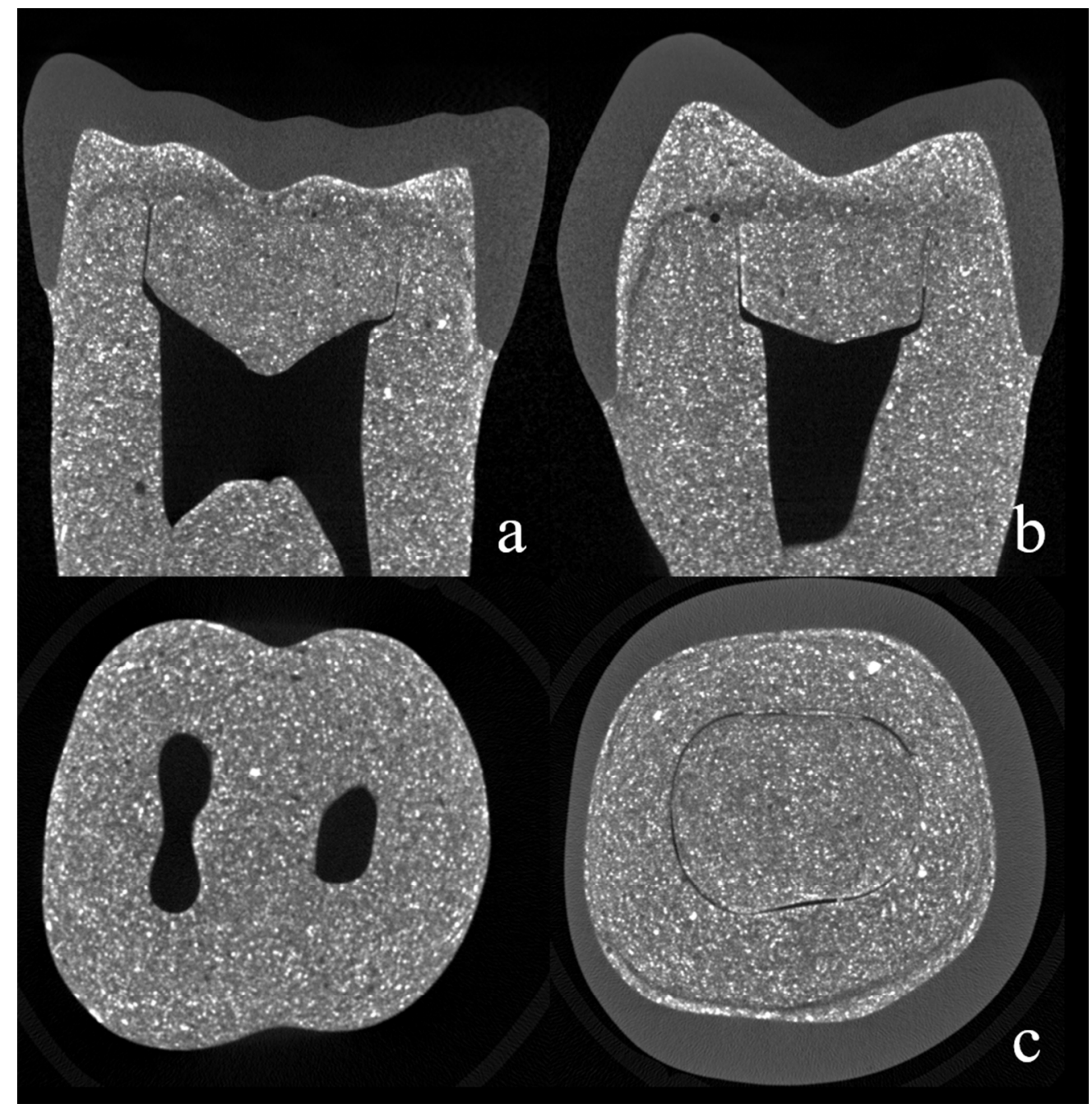

Fig. 2. Slices of artificial mandibular first molar (a) mesiodistal section, exposing the buccal and lingual aspect of the pulp cavity, (b) buccolingual section, exposing the medial and distal aspect of the pulp cavity, (c) transverse section trunk and occlusal.

$176 \times 180 \mathrm{~mm}(150 \times 150 \mathrm{DPI})$ 


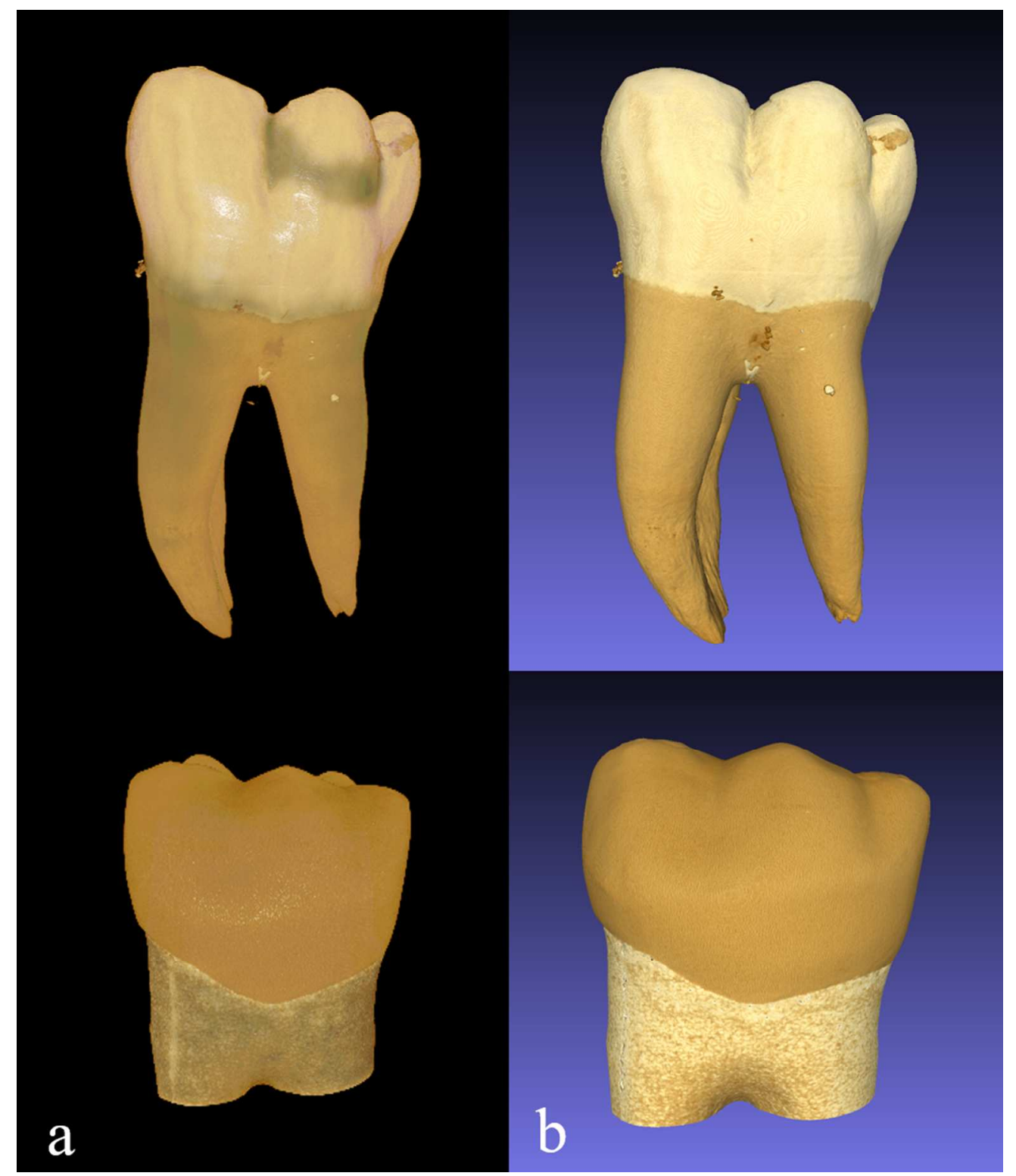

Fig. 3. Rendered surface images of both natural and artificial mandibular first molar (a) viewed in Drishti, (b) viewed in Meshlab.

$174 \times 206 \mathrm{~mm}(150 \times 150 \mathrm{DPI})$ 


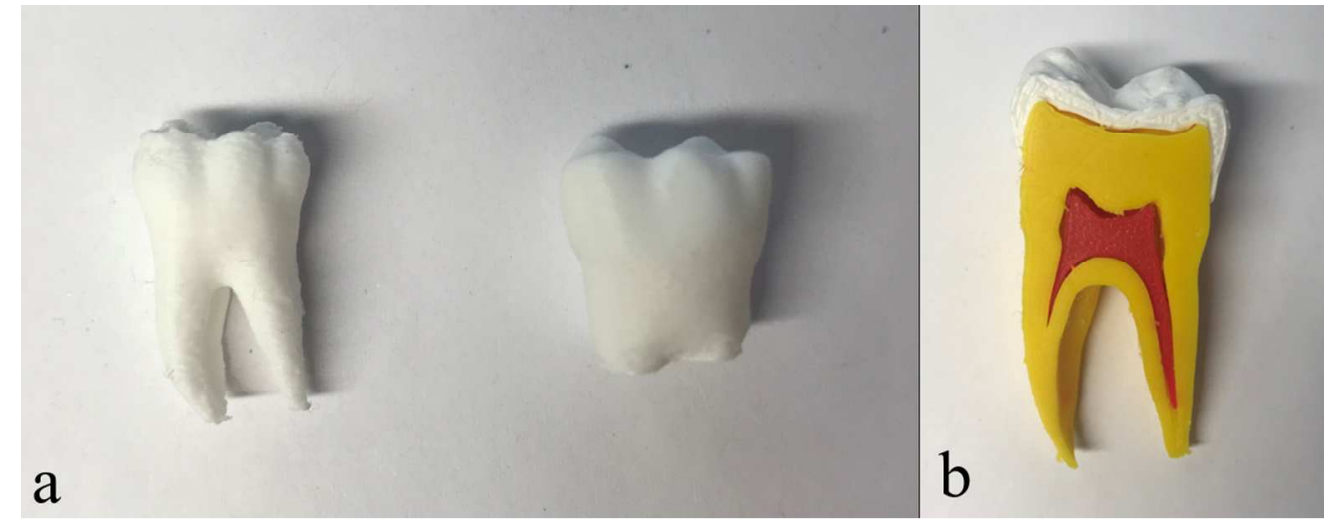

Fig. 4. 3D printed mandibular first molar (a) both natural and artificial molar in PLA, (b) natural molar in multiple materials, PLA for enamel, TPE for dentine and pulp cavity.

\section{$222 \times 87 \mathrm{~mm}(150 \times 150 \mathrm{DPI})$}




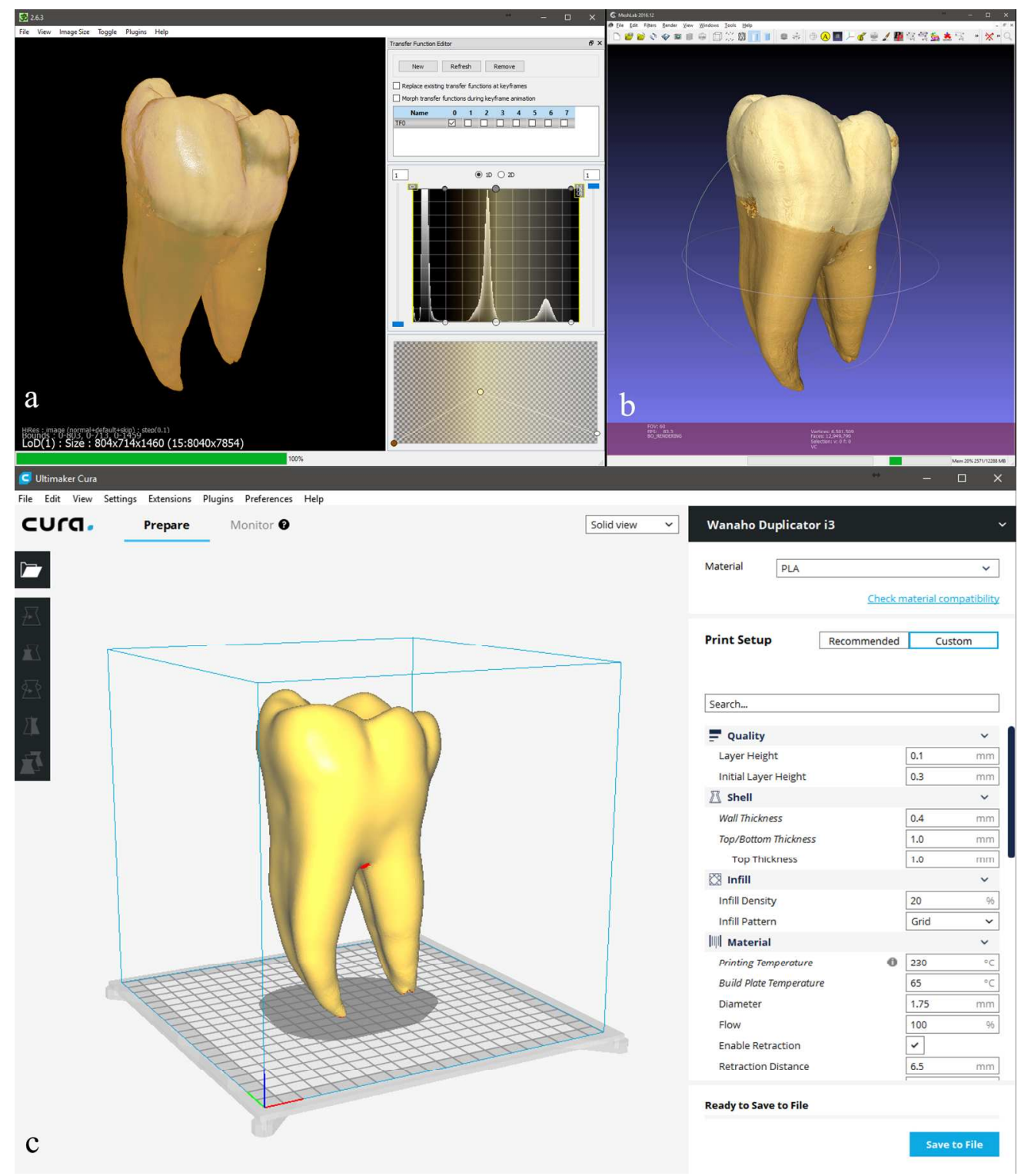

Fig. 5. Screenshots of the open-source software used to convert tomography files to 3D print (a) rendering of 3D surface in Drishti, (b) rendering of 3D surface in Meshlab, with the ability to manipulate file to desired final product, (c) slicing view in Cura, with the ability to edit layer settings. 
Objectives: Trainee dentists practice procedures using artificial teeth that are far from real teeth. Using x-rays and 3D printing technology the project will re-create a real tooth, artificially.

Methods: X-rays produce a 3D image that can be printed out as a physical replica, after several conversions of files. Different settings can be used to allow the printed model, to be as accurate as possible. Data was collected on the forces from a dental drill on a tooth's surface, to measure hardness and resistance.

Results: Multiple teeth replicas were printed with a high accuracy. The materials printed did not mimic actual tooth properties, but using the data from real teeth, materials can be tested in future. 